\title{
A simple method for correcting single breath total lung capacity for underestimation
}

Alain Loiseau, Paule Loiseau, Georges Saumon

\begin{abstract}
The single breath method underestimates total lung capacity by comparison with the multiple breath method (TLCmb) because of inhomogeneity of ventilation distribution. This study proposes a simple correction for the single breath TLC (TLCsb), using inert gas phase III slope to account for the effects of uneven ventilation distribution. A model of a non-uniform lung ventilation was designed, composed of a serial dead space and two alveolar compartments arranged in parallel, whose relative ventilations were determined from the phase III plateau. Before correction TLCsb was $104-44 \%$ of TLCmb in 64 subjects (17 with diffuse interstitial disease, 42 with chronic obstructive pulmonary disease, and five healthy subjects). The limit of acceptability for the correction (TLCcorr) was determined from the $95 \%$ confidence interval of TLCsb/TLCmb in the healthy subjects. The correction resulted in a significant increase in TLCsb $(p<0.004)$. TLCcorr remained under the limit of acceptability for only 12 patients with emphysema, and all 12 showed a large improvement in the TLC estimate. The presence of poorly ventilated zones during a single breath in these patients may explain this partial correction.
\end{abstract}

The single breath inert gas dilution test underestimates total lung capacity (TLC) by comparison with the closed circuit multibreath technique. This underestimation parallels the uneven distribution of ventilation in patients. ${ }^{1-5}$ Although an inspired inert gas (such as helium or argon) is distributed almost evenly to all areas of the lung that contribute to the single breath phase III in healthy subjects, this is far from being the case in patients. The distribution of volume and ventilation is often very uneven in patients with lung disease, ${ }^{6}$ leading to a mean expired inert gas concentration that is larger than would be expected from simple dilution. This is due partly to the fact that the mean gas concentration that results from the mixing of different compartments with their own dilution and ventilation ratios is always larger than the mean concentration obtained when all compartments have the same dilution ratio (but different volumes) or the same ventila- tion ratio. ${ }^{3}$ Furthermore, the asynchronous filling and emptying of these lung regions follows various regional or local unpredictable patterns. A remarkable consequence of these different uneven patterns is reflected by the slope of the expired inert gas concentration curve (the so called phase III plateau). Many studies have shown that the slope of the phase III plateau or some of its derivatives are related to pulmonary abnormalities present in various lung diseases. ${ }^{7}$ Corrections have been proposed to provide a better estimate of TLC during a single breath test. ${ }^{48}$ They are based on the observation of a statistically significant relation between the underestimation of TLC and an explicative variable obtained from lung function testing $\left(\mathrm{FEV}_{1} / \mathrm{VC}^{8} ; \mathrm{N}_{2}\right.$ slope of phase III plateau after a single breath of oxygen ${ }^{4}$ ).

Our objective was to propose a simple correction for underestimation of the single breath TLC based on the slope of the inert gas phase III plateau. Considering that this slope is the consequence of ventilation inhomogeneities (either in series or in parallel $^{9-16}$ ), we designed a model of uneven lung filling and emptying, composed of two alveolar compartments with opposite characteristics. The ventilation:volume ratio of each compartment was deduced from the phase III plateau. This model provided a new (corrected) residual volume and thus a corrected TLC; this was then compared with the multibreath closed circuit helium dilution TLC in a sample population of healthy subjects and patients. Multibreath TLC was chosen as a reference rather than radiographic or plethysmographic TLC because it represents ventilated lung (effective volume). The proposed correction can be performed with a pocket calculator.

\section{Methods}

\section{STUDY POPULATION}

Four groups of subjects were studied: five healthy subjects, 17 patients with interstitial lung disease of various origins, 10 patients with chronic obstructive lung disease without radiological evidence of pulmonary emphysema, and 32 patients with radiological evidence of emphysema. Healthy subjects were non-smokers and had no previous history of respiratory disease. Patients with chronic obstructive lung disease and most of those with emphysema were heavy smokers. Patients with interstitial lung disease showed different stages of pulmonary disease, ranging from slight to fibrotic. Physical and lung function data for the 
Physical and functional values (mean (SD)) in the subjects

\begin{tabular}{|c|c|c|c|c|c|c|c|c|}
\hline & $\begin{array}{l}\text { Sex } \\
(F: M)\end{array}$ & $\begin{array}{l}\text { Age } \\
(y)\end{array}$ & $\begin{array}{l}\text { Height } \\
(\mathrm{cm})\end{array}$ & $\begin{array}{l}\text { Weight } \\
(\mathrm{kg})\end{array}$ & $\begin{array}{l}V C^{\star} \\
(\%)\end{array}$ & $\begin{array}{l}R V / T L C^{\star} \dagger \\
(\%)\end{array}$ & $\begin{array}{l}F E V_{1} / V C^{\star} \\
(\%)\end{array}$ & $\begin{array}{l}\alpha A r \\
\left(\% l^{-1}\right)\end{array}$ \\
\hline $\begin{array}{l}\text { Healthy }(n=5) \\
\text { Interstitial lung disease }(n=17) \\
\text { Chronic obstructive lung disease }(n=10) \\
\text { Emphysema }(n=32)\end{array}$ & $\begin{array}{l}1: 4 \\
6: 11 \\
1: 9 \\
4: 28\end{array}$ & $\begin{array}{l}38(9 \cdot 9) \\
38(15 \cdot 5) \\
55(13 \cdot 1) \\
51(13 \cdot 1)\end{array}$ & $\begin{array}{l}170(14 \cdot 1) \\
168(7 \cdot 3) \\
174(6 \cdot 6) \\
169(7 \cdot 5)\end{array}$ & $\begin{array}{l}68(9 \cdot 8) \\
64(9 \cdot 0) \\
79(16 \cdot 1) \\
62(13 \cdot 6)\end{array}$ & $\begin{array}{l}92.0(11 \cdot 1) \\
93.5(17.6) \\
83.0(14.3) \\
86.0(23.0)\end{array}$ & $\begin{array}{r}107(9 \cdot 4) \\
91(19 \cdot 8) \\
111(24 \cdot 7) \\
149(32 \cdot 4)\end{array}$ & $\begin{array}{r}113(4 \cdot 9) \\
93(10 \cdot 7) \\
63(24 \cdot 4) \\
51(21 \cdot 7)\end{array}$ & $\begin{array}{l}-0.8(0.38) \\
-2.3(1.78) \\
-4.7(2.67) \\
-9.9(6.27)\end{array}$ \\
\hline
\end{tabular}

$\star$ Percentage of predicted value.

$\dagger R V$ was measured by multibreath helium dilution; TLC $=V C+R V$ multibreath.

$\alpha \mathrm{Ar}-$ Argon slope of the single breath phase III.

subjects are presented in the table. The predicted values for lung function are those of Quanjer et al. ${ }^{17}$

\section{MEASUREMENTS}

Lung volumes and $\mathrm{FEV}_{1}$ were measured with a water sealed spirometer (Godart, Bilthoven, The Netherlands). Residual volume ( $R V m b$ ) was measured by the usual multibreath helium dilution technique. The inert gas single breath test was performed in duplicate, with a gas mixture containing $79 \%$ argon and $21 \%$ oxygen. The subject exhaled until residual volume was attained, inspired slowly to attain total lung capacity, and then exhaled slowly back to residual volume. Two signals were monitored: mouth flow was determined by means of a Fleisch No 1 pneumotachograph and a $\pm 0.2 \mathrm{kPa}$ transducer (MP45, Validyne, Northridge, California), and the argon concentration was sampled at the mouth to feed a mass spectrometer (MS4, AEI, Manchester, England). These signals were digitised at a sampling rate of $125 \mathrm{~Hz}$ and stored on magnetic disk. They were synchronised using the time response of the spectrometer $(95 \%$ in $130 \mathrm{~ms})$ before subsequent calculations were performed with a System 1000 Hewlett-Packard computer (Hewlett-Packard, Palo Alto, California).

The slope and intercept at zero (TLC) expired volume of the phase III argon plateau as a function of expired volume were calculated by linear regression. The calculation was per-
RV

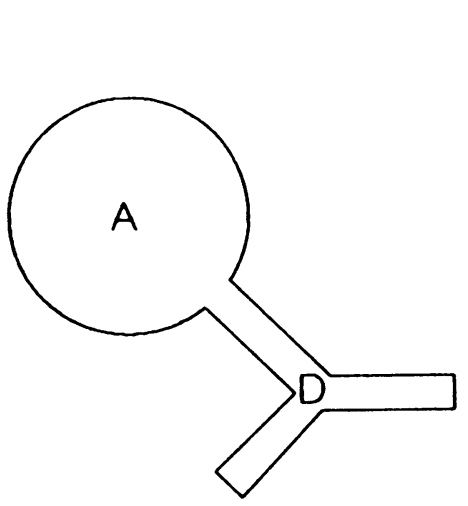

TLC

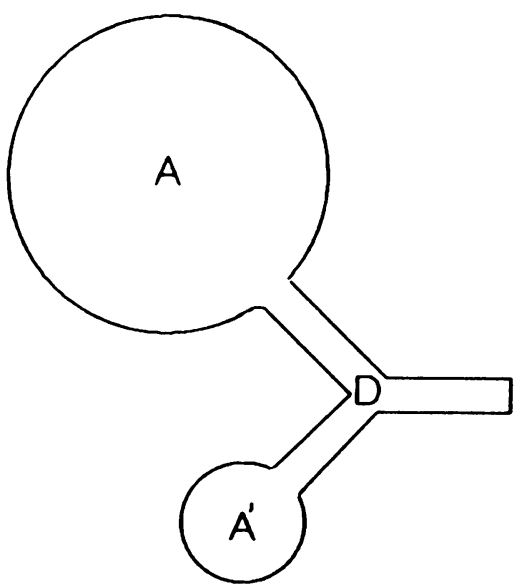
Figure 1 Schematic representation of the model. $D$-serial dead space; $A-$ gas
exchanging alveolar compartment; $A^{\prime}-$ alveolar dead space. At residual volume the $A^{\prime}$ compartment is empty; it is filled progressively as lung expands with inspired gas. formed between the end of the deadspace washout and the closing volume (when present), both being recognised by eye. Subsequent calculations are provided in detail in the appendix. Briefly, the single breath TLC (TLCsb) and residual volume (RVsb) were calculated using the usual equation of dilution, by numerical integration of the product of mouth flow and argon concentration. Appropriate corrections were made for instrumental $\left(50 \mathrm{~cm}^{3}\right)$ and anatomical deadspaces, and for the background argon concentration $\left(1^{\circ}{ }_{0}\right)$. The corrected residual volume (RVcorr) was obtained from a model of lung ventilation whose characteristics are shown in figure 1. It consisted of three gas compartments, $D, A$, and $A^{\prime} . D$ is the usual serial deadspace, though $A$ and $A^{\prime}$ are alveolar compartments arranged in parallel. At residual volume the volume of compartment $A$ was $\mathrm{RV}$ corr, and that of compartment $\mathrm{A}^{\prime}$ was zero. This choice was imposed by the number of parameters to be identified, and was limited by the amount of information available from the single breath phase III. During inspiration, after the alveolar air from the preceding breath in $D$ had returned to $A$, the mouth flow was partitioned between $A$ and $A^{\prime}$. This partition was chosen among the most simple ones: it was a linear function of the changing lung volume. At the beginning of inspiration $A$ received all the flow entering the mouth. Then less and less flow filled $A$ as the lung expanded, and the remaining flow went into $A^{\prime}$, which was consequently filled with the pure inspired mixture. This procedure was repeated in reverse during expiration. The rationale for this choice was that it represented an oversimplification of possible mechanisms for inhomogeneous lung filling and emptying: the upper lung regions with the largest $R V$ filled first, then became too rigid to receive the bulk of inspired flow, allowing filling of more compliant ones. ${ }^{1819}$ The partition of ventilation between $A$ and $A^{\prime}$ was derived from the slope of the phase III plateau $(\alpha)$ : the steeper $\alpha$ was the larger the volume $A^{\prime}$ received. From the alveolar plateau we could calculate the parameter of flow partition, RVcorr, and TLCcorr.

\section{STATISTICAL METHODS}

Comparisons were made with the paired $t$ test and the statistical significance level was set at $<0.05$. Linear regressions and correlations were performed by the least mean squares method. 
Figure 2 Graph of TLCsb and TLCcorr plotted against TLCmb. The solid line represents the line of identity and the broken line the $95 \%$ lower limit for acceptability of the TLC correction. normal; $\bigcirc$ interstitial lung disease; chronic obstructive lung disease; $\square$ emphysema.
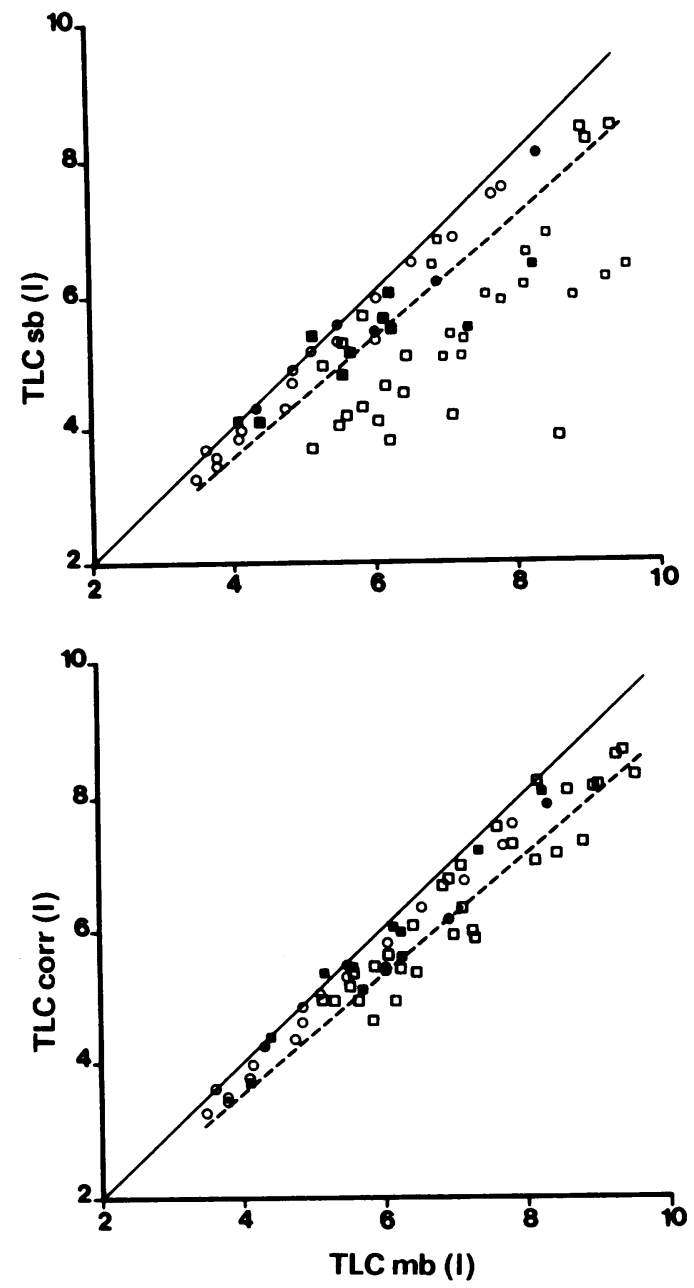

Results

The study groups differed in age, airway obstruction (present in patients with chronic obstructive lung disease and emphysema), RV/TLC ratio (increased in group with emphysema), and ventilation distribution (an abnormal $\alpha$ value in all patients, with a steep slope in patients with chronic bronchitis and a steeper slope in those with emphysema: table). The subjects displayed a wide range of abnormal results and, as expected, TLCsb was underestimated by comparison with TLCmb in almost all patients (fig 2, top). The ratio TLCsb/TLCmb ranged from $104 \%$ to $44 \%$. No significant difference was observed between the two estimates in the healthy subjects. The variability of TLCsb/TLCmb in these patients enabled us to define the limit (from the $95 \%$ confidence interval) for accepting TLCcorr as a good estimate of TLCmb. This limit was equal to $89 \%$ of TLCmb (fig 2, top). This variability is similar to that found in previous studies with a larger number of subjects. ${ }^{2021}$ TLCsb was below this value in 28 of the 64 subjects (one with interstitial lung disease, four with chronic obstructive lung disease, 23 with emphysema).

The effect of our proposed correction to TLCsb is illustrated in figures 2 (bottom) and 3. There was no significant difference between TLCcorr and TLCsb for the subjects in whom TLCsb correctly estimated TLCmb. For the
28 remaining patients the correction was significantly different from zero $(p<0.004)$. TLCcorr remained below the limit of acceptability in 12 of the 28 patients, all of whom had emphysema. The correction nevertheless improved the estimate of TLCsb, TLCsb increasing from $72 \%$ of TLCmb (minimum $60.5 \%$ ) to $83.6 \%$ (minimum $79 \%$ ) in these patients. There was no difference in $\mathrm{VC}, \mathrm{FEV}_{1} /$ VC, RV/TLCmb, or the $\alpha$ value between patients with well corrected or poorly corrected TLC.

\section{Discussion}

METHODOLOGICAL CONSIDERATIONS

Non-uniform ventilation distribution may be explained by the interaction of multiple factors: differences in time constants from one lung region to another, ${ }^{19}$ an apicocaudal gradient of pleural pressure, ${ }^{18}$ bronchial closure at low volumes, ${ }^{12223}$ and local differences in acinar anatomy. ${ }^{23}$ All of these factors are likely to affect non-uniform distribution, whether in series (diffusion distances) or in parallel (differences in $\dot{V}_{A} / V_{A}$, asynchronous filling and emptying). We did not attempt to take these events into account to obtain a correction for the single breath TLC measurement. Firstly, it appeared impossible to model accurately a process that includes so many parameters and unknown elements, especially in patients. Secondly, as our work would eventually result in identification of the parameters of a model of ventilation there could not be more than two of these because only two values could be obtained from the single breath phase III plateau (that is, slope and intercept). From the various possibilities, we chose a model composed of two alveolar compartments arranged in parallel and two parameters, the residual volume and the ratio of ventilation between the two compartments. For the phase III slope to be mimicked, however, asynchronous ventilation is necessary. The choice of a linear decrease as a function of lung volume for the relative ventilation of the $A$ compartment was made for the sake of simplicity. We tried other patterns of ventilation distribution (that is, exponential), but without further success in improving poorly corrected values.

Obviously, any partition between the residual volumes of the $A$ and $A^{\prime}$ compartments might be imposed but unfortunately might not be identified. A zero residual volume for $\mathrm{A}^{\prime}$ was not a purely arbitrary choice. It corresponded to the proportion of inspired air that would never mix with residual gas. A possible physical analogue for this would be diffusion inhomogeneity, as a central core of gas penetrating alveoli would increase in volume during inspiration but only slowly (or even neverthe extreme hypothesis) $\mathrm{mix}$ with residual gas (fig 1). Any other type of non-uniform distribution would, however, still be represented by a model with two compartments in parallel, as distinguishing between series or parallel inhomogeneities is impossible because their effects on expired gas concentration are identical. ${ }^{9-11}$ 
Helium is frequently used as an inert gas for TLC measurement. The derivation of the correction equation was made with argon. Whether helium and argon provide the same TLCsb estimates requires confirmation. The phase III slope is almost certainly steeper for argon than for helium because of its lesser diffusivity. ${ }^{24}$ Phase III slope is not, however, the only parameter of the alveolar plateau used for correction of TLC. The intercept of inert gas concentration at zero volume is also a determinant of this correction. Then the proposed correction (after simplification for helium because of the zero alveolar concentration) remains to be validated when an inert gas other than argon is used.

\section{RELATION WITH MALDISTRIBUTION OF VENTILATION}

The uneven distribution of ventilation is of mechanical origin. ${ }^{181922}$ The difference in time constants between lung regions is the result of gravity, ${ }^{91819}$ uneven distribution within regions, ${ }^{11} 23$ and diffusion processes. ${ }^{10}$ In disease lung lesions also contribute to maldistribution. ${ }^{67}$ Thus attempts at modelling lung function in patients are futile. The gross characteristics may be the subject of phenomenological oversimplification, and such is the case here. The origin of the underestimation of TLC is likely to differ from one patient to another one. As, however, all patients' TLCsb values were corrected (though insufficiently in some cases), we consider that the proposed correction mirrors to some extent existing uneven distribution of ventilation. The basis of the correction is that zones with the largest $R V$ must fill first. The patterns of filling and of further emptying are entirely arbitrary, and we do not have any additional experimental evidence to prove this. So we are aware that this is a representative rather than a comprehensive model.

The insufficiently corrected TLC values were, however, those of emphysematous patients only. We propose as an explanation that in these patients quasi unventilated areas are present during the single breath test (areas with very large time constants), which did not receive enough inspired gas to affect the single breath plateau significantly, and therefore could not be corrected on the basis of the phase III slope. These lung regions are more or less ventilated during rebreathing, ${ }^{25}$ accounting for the remaining difference between TLCcorr and TLCmb.

Further work is needed to determine whether the correction equation applies to larger flow rates, breath holding of various durations, and helium.

\section{We wish to thank Ms F Miklovic for typing the manuscript.}

\section{Appendix: Calculations for correcting single breath total lung capacity}

This model consists of a serial dead space (D) and two homogeneous parallel alveolar spaces: an alveolar compartment (that would eventually participate in gas exchange) (A) and an alveolar dead space $\left(A^{\prime}\right)$. The volume of the serial compartment (VD) was calculated from the total lung volume (TLC) by the equation of Martin et al..$^{26}$ To simplify calculations it was considered as a constant volume.

To describe the single breath phase III for argon a changing distribution of ventilation in the $A$ and $A^{\prime}$ compartments as a function of inspired (I) or expired (E) volume was imposed according to the following equations:

$$
\begin{aligned}
& \dot{\mathrm{V}} \mathrm{A}^{\prime} \mathrm{I}(\mathrm{t}) / \dot{\mathrm{V} m I}(\mathrm{t})=\mathrm{aVmI}(\mathrm{t}) \\
& \dot{\mathrm{V}} \mathrm{A}^{\prime} \mathrm{E}(\mathrm{t}) / \dot{\mathrm{V} m \mathrm{~m}}(\mathrm{t})=\mathrm{a}[\mathrm{VCI}-\operatorname{VmE}(\mathrm{t})],
\end{aligned}
$$

where $(\mathrm{VCI})$ is the inspiratory vital capacity, $(\dot{\mathrm{V} m})$ and $(\mathrm{Vm})$ are mouth flow and volume, and (a) is the flow partition parameter to be estimated. The inflow and outflow for the A space was obtained by subtraction:

$$
\dot{\mathrm{V}} \mathrm{A}(\mathrm{t})=\dot{\mathrm{V}} \mathrm{m}(\mathrm{t})-\dot{\mathrm{V}}_{\mathrm{A}^{\prime}}(\mathrm{t})
$$

The initial conditions were fractional argon concentrations and lung volumes for $\mathrm{A}$ and $\mathrm{A}^{\prime}$ compartments: $\mathrm{VA}_{0}=\mathrm{RV}-\mathrm{VD} ; \mathrm{FA}_{0}=1 \%$ and $\mathrm{VA}_{0}^{\prime}=0$, where $\mathrm{RV}$ (residual volume) is the second parameter to be estimated.

By integration of the flow equations, the $A^{\prime}$ inspired and expired volumes are obtained:

$$
\begin{aligned}
& V_{A^{\prime}} I(t)=a \operatorname{lmI}^{2}(\mathrm{t}) / 2 \\
& \mathrm{VA}^{\prime} \mathrm{E}(\mathrm{t})=\mathrm{a}[\mathrm{VCI}-\operatorname{VmE}(\mathrm{t})]^{2} / 2 .
\end{aligned}
$$

The values of the compartments at TLC are:

$$
\begin{aligned}
& V_{A^{\prime}} \max =\mathrm{aVCI}^{2} / 2 \\
& V_{A m a x}=\mathrm{VCI}+\mathrm{RV}-\mathrm{VD}-\mathrm{VA}^{\prime} \max .
\end{aligned}
$$

At the end of the inspiration the argon present in compartments $A$ and $A^{\prime}$ is equal to the sum of the residual and incoming quantities:

$$
\begin{aligned}
& F_{A^{\prime}} V_{A}^{\prime} \max =\text { FIVA'max } \\
& \left(F_{A^{\prime}}=\text { FI because RV of } A^{\prime} \text { is equal to } 0\right) \\
& \text { FAVAmax }= \\
& \quad \text { FI }\left(V_{C I}-V_{A}^{\prime} \max -V D\right)+R V .
\end{aligned}
$$

During expiration the gas that issued from $A$ and $A^{\prime}$ mixes in proportion to the respective ventilation ratios. The expired argon concentration in serial dead space compartment $(\mathrm{FE}(\mathrm{t}))$ is:

$$
\operatorname{FE}(t)=[\operatorname{FA} \dot{V} A E(t) / \dot{V} m E(t)]+
$$$$
\left[F_{A^{\prime}} \dot{V}^{\prime} \mathrm{E}(\mathrm{t}) / \dot{\mathrm{V}} \mathrm{mE}(\mathrm{t})\right] \text {. }
$$

When equations 2 and 3 are substituted into equation 10 and $\mathrm{FE}$ is expressed as a function of expired volume (VmE), the abscissa of the plot used to perform the linear regression of the inert gas single breath phase III plateau is:

$$
\begin{array}{r}
\mathrm{FE}(\mathrm{VmE})=\mathrm{FA}_{\mathrm{A}}\left[1-\mathrm{a}\left(\mathrm{VCI}_{\mathrm{I}}-\mathrm{VmE}\right)\right]+ \\
\mathrm{aFA}^{\prime}(\mathrm{VCI}-\mathrm{VmE}) .
\end{array}
$$

This argon concentration appears at the mouth $(\mathrm{FmE})$ after a transit time corresponding to the transport across the serial dead space. This correspondence, expressed as a function of expired volume, is:

$$
\mathrm{FE}(\mathrm{vmE})=\mathrm{FmE}(\mathrm{VmE}+\mathrm{VD}) .
$$

Let $\alpha$ be the slope and $\beta$ the concentration intercept at zero expired volume of the phase III plateau. Then the phase III argon concentration measured at the mouth, expressed as a function of expired volume, is:

$\mathrm{FmE}(\mathrm{VmE})=\alpha \mathrm{VmE}+\beta$

$\mathrm{FmE}(\mathrm{VmE}+\mathrm{VD})=\alpha(\mathrm{VmE}+\mathrm{VD})+\beta$.

Equation 12 becomes:

$$
\mathrm{FE}(\mathrm{VmE})=\alpha(\mathrm{VmE}+\mathrm{VD})+\beta
$$

$\mathrm{FE}(\mathrm{VmE})$ is replaced by its value in equation 11 , which is rearranged as:

$$
\begin{aligned}
& \mathrm{a}\left(\mathrm{FA}_{\mathrm{A}}-\mathrm{FA}^{\prime}\right) \mathrm{VmE}+\mathrm{FA}_{\mathrm{A}}(1-\mathrm{aVCI})+ \\
& \mathrm{aFA}^{\prime} \mathrm{VCI}=\alpha \mathrm{VmE}+\alpha \mathrm{VD}+\beta .
\end{aligned}
$$

This equation is equivalent to the system:

$$
\begin{aligned}
& \mathrm{a}\left(\mathrm{FA}_{\mathrm{A}}-\mathrm{FA}^{\prime}\right)=\alpha \\
& \quad \text { (dividing both sides by } \mathrm{VmE})
\end{aligned}
$$

$$
\mathrm{FA}(1-\mathrm{aVCI})+\mathrm{aFA}^{\prime} \mathrm{VCI}=\alpha \mathrm{VD}+\beta . \quad \text { (17) }
$$

The substitution of $F_{A}$ and $F_{A}^{\prime}$ by their expressions 
in equations 8 and 9 and the replacement of $\mathrm{VA}^{\prime} \max$ by its value in equation 6 and VAmax by equation 7 into the system 16 and 17 produces:

$$
\begin{aligned}
& -\alpha \mathrm{aVCI}^{2} / 2+\mathrm{aRV}\left(\mathrm{FI}_{\mathrm{I}}-1\right)+ \\
& \alpha \mathrm{RV}+\alpha(\mathrm{VCI}-\mathrm{VD})=0 \\
& -\mathrm{aVCI}^{2}\left(\mathrm{FI}^{-}-\alpha \mathrm{VD}-\beta\right) / 2+ \\
& \mathrm{aRVVCI}(\mathrm{FI}-1)+\mathrm{RV}(1-\alpha \mathrm{VD}-\beta)+ \\
& \quad(\mathrm{VCI}-\mathrm{VD})\left(\mathrm{FI}_{\mathrm{I}}-\alpha \mathrm{VD}-\beta\right)=0 .
\end{aligned}
$$

This system is solved by multiplying equation 19 by $(-\alpha)$ and equation 18 by $(\mathrm{FI}-\alpha \mathrm{VD}-\beta)$ and summation. It becomes:

$$
\begin{array}{r}
\mathbf{R V}\left(\mathrm{FI}_{\mathbf{I}}-1\right) \\
\left\{\alpha+\mathrm{a}\left[\mathrm{FI}_{\mathbf{I}}-\alpha(\mathrm{VCI}+\mathrm{VD})-\beta\right]\right\}=0,
\end{array}
$$

$\left(F_{I}-1\right)$ and $(R V)$ cancel because they are different from zero; then:

$$
\mathrm{a}=-\alpha /[\mathrm{FI}-\alpha(\mathrm{VCI}+\mathrm{VD})-\beta] .
$$

The corrected $\mathrm{RV}$ is obtained replacing this expression for (a) into equation 18, and subtracting the instrumental dead space volume.

RVcorr =

$\left\{(\mathrm{VCI}-\mathrm{VD})\left[\mathrm{FI}_{\mathrm{I}}-\alpha(\mathrm{VCI}+\mathrm{VD})-\beta\right]+\alpha \mathrm{VCI}^{2} / 2\right\} /$
$[\alpha(\mathrm{VCI}+\mathrm{VD})+\beta-1]-50 .(21)$

Then from the four measurements $\mathrm{FI}_{\mathrm{I}}, \mathrm{VCI}, \alpha, \beta$, and one derived value, VD, TLCcorr can be easily computed: $\mathrm{TLC}$ corr $=\mathrm{VCI}+\mathrm{RV}$ corr .

When helium is used as inert gas, the denominator of equation 21 is simplified because of the zero alveolar concentration. It becomes $\alpha(\mathrm{VCI}+\mathrm{VD})+\beta$.

1 Buist AS, Ross BB. Predicted values for closing volume using a modified single breath nitrogen test. Am Rev Respir Dis 1973;107:744-52.

2 Morton JW, Ostensoe LG. A clinical review of the single breath method of measuring the diffusing capacity of the lungs. Dis Chest 1965;48:44-54.

3 Piiper J, Sikand RS. Determination of Dco by the single breath method in inhomogeneous lung. Theory Respir Physiol 1966;1:75-87.

4 Sterk PJ, Quanjer Ph, Van der Maas LLJ, Wise ME, Van Lende $R$. The validity of the single breath nitrogen determination of residual volume. Bull Eur Physiopathol Respir 1980;16:195-213.

5 Teculescu DB, Stanescu DC. Total lung capacity in obstructive lung disease comparative determinations by single and multiple-breath helium dilution. Bull Eur Physiopathol Respir 1969;5:453-64.

6 Hughes JMB, Amis TC. Regional ventilation distribution. In: Engel LA, Paiva M, eds. Gas mixing and distribution in the lungs. New York: Dekker, 1985:177-212.
7 Engel LA, Farhi LE. Airway closure, closing volume, and changes in inert gas mixing. In: Macklem PT, Permutt $S$, eds. The lung in the transition between health and disease. New York: Dekker, 1979:153-82.

8 Burns CB, Scheinhorn DJ. Evaluation of single breath helium dilution total lung capacity in obstructive lung disease. Am Rev Respir Dis 1984;130:580-3.

9 Anthonisen NR, Robertson PC, Ross WRD. Gravitydependent sequential emptying of lung regions. $J$ Appl Physiol 1970;28:589-95.

10 Cumming G, Horsfield K, Jones JG, Muir DCF. The influence of gaseous diffusion on the alveolar plateau at different lung volumes. Respir Physiol 1967;2:386-98.

11 Engel LA, Utz G, Wood LDH, Macklem PT. Ventilation distribution in anatomical lung units. J Appl Physiol 1974;37:194-200.

12 Fowler WS. Lung function studies III. Uneven pulmonary ventilation in normal subjects and in patients with pulmonary disease. $J$ Appl Physiol 1949;2:283-99.

13 Hook C, Meyer M, Piiper J. Model simulation of single breath washout of insoluble gases from dog lungs. $J$ Appl Physiol 1985;58:802-11.

14 Nixon W, Pack AI. Effect of gaseous interaction between lung units on the expired concentration of nitrogen. $J$ Appl Physiol Respirat Environ Exercise Physiol 1982;53: 496-504.

15 Paiva M, Engel LA. Pulmonary interdependence of gas transport. J Appl Physiol Respirat Environ Exercise Physiol 1979;47:296-305.

16 Paiva M, Engel LA. The anatomical basis for the sloping $\mathrm{N}_{2}$ plateau. Respir Physiol 1981;44:325-37.

17 Quanjer $\mathrm{Ph}$, ed. Standardized lung function testing. European community for Coal and steel. Bull Eur Physiopathol Respir 1983;19(suppl 5):45-51.

18 Milic-Emili J, Henderson JAM, Dolovich MB, Trop D, Kaneko K. Regional distribution of inspired gas in the lung. J Appl Physiol 1966;21:749-54.

19 Otis AB, McKerrow CB, Bartlett A, et al. Mechanical factors in distribution of pulmonary ventilation. J Appl Physiol 1956;8:427-43.

20 Lyons JP, Clarke WC, Hall AM, Cotes JE. Transfer factor (diffusing capacity) for the lung in simple pneumoconiosis of coal markers. Br Med J 1967;ii:772-4.

21 Teculescu DB. Validity, variability and reproducibility of single breath total lung capacity determinations in normal subjects. Bull Physiopathol Respir 1971;7:645-58.

22 Dollfuss RE, Milic-Emili J, Bates DV. Regional ventilation of the lung studied with boluses of ${ }^{133}$ xenon. Respir Physiol 1967;2:234-46.

23 Horsfield $\mathrm{K}$. Anatomical factors influencing gas mixing and distribution. In: Engel LA, Paiva M, eds. Gas mixing and distribution in the lungs. New York: Dekker, 1985:23-61.

24 Engel LA. Intraregional gas mixing and distribution. In: Engel LA, Paiva M, eds. Gas mixing and distribution in the lung. New York: Dekker, 1985:287-358.

25 Medina JR, Lillehei JP, Loken MK, Ebert RV. Use of the scintillation anger camera and xenon 133 in the study of chronic obstructive lung disease. JAMA 1969;208: 985-91.

26 Martin CJ, Das S, Young AC. Measurements of the dead space volume. J Appl Respir Environ Exercise Physiol 1979;47:319-24. 